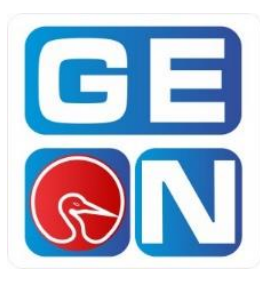

Revista GEON (Gestión, Organizaciones y Negocios.) ISSN: 2346-3910 en línea

revistageon@unillanos.edu.co

Universidad de los Llanos

Colombia

Pérez Paredes, Alfredoi; Cruz de los Ángeles, José Aurelioii;

Guatemala Villalobos, Araceli Ma. De Jesús siii ; Juárez Fonseca,

Verónica $^{\text {iv }}$.

\title{
Importancia de los pronósticos en la toma de decisiones en las MIPYMES
}

Revista GEON, Vol. 5, No. 1, 2018

Pág. 97-114

Disponible en: https://doi.org/10.22579/23463910.17

\footnotetext{
${ }^{\mathrm{i}}$ https://orcid.org/0000-0001-8766-5766

ii https://orcid.org/0000-0002-8682-366X

iii https://orcid.org/0000-0001-7323-6848

iv $\underline{\text { https://orcid.org/0000-0002-0562-1063 }}$
}

Esta publicación se encuentra bajo licencia: Creative Commons

ReconocimientoNoComercialSinObraDerivada 4.0 Internacional
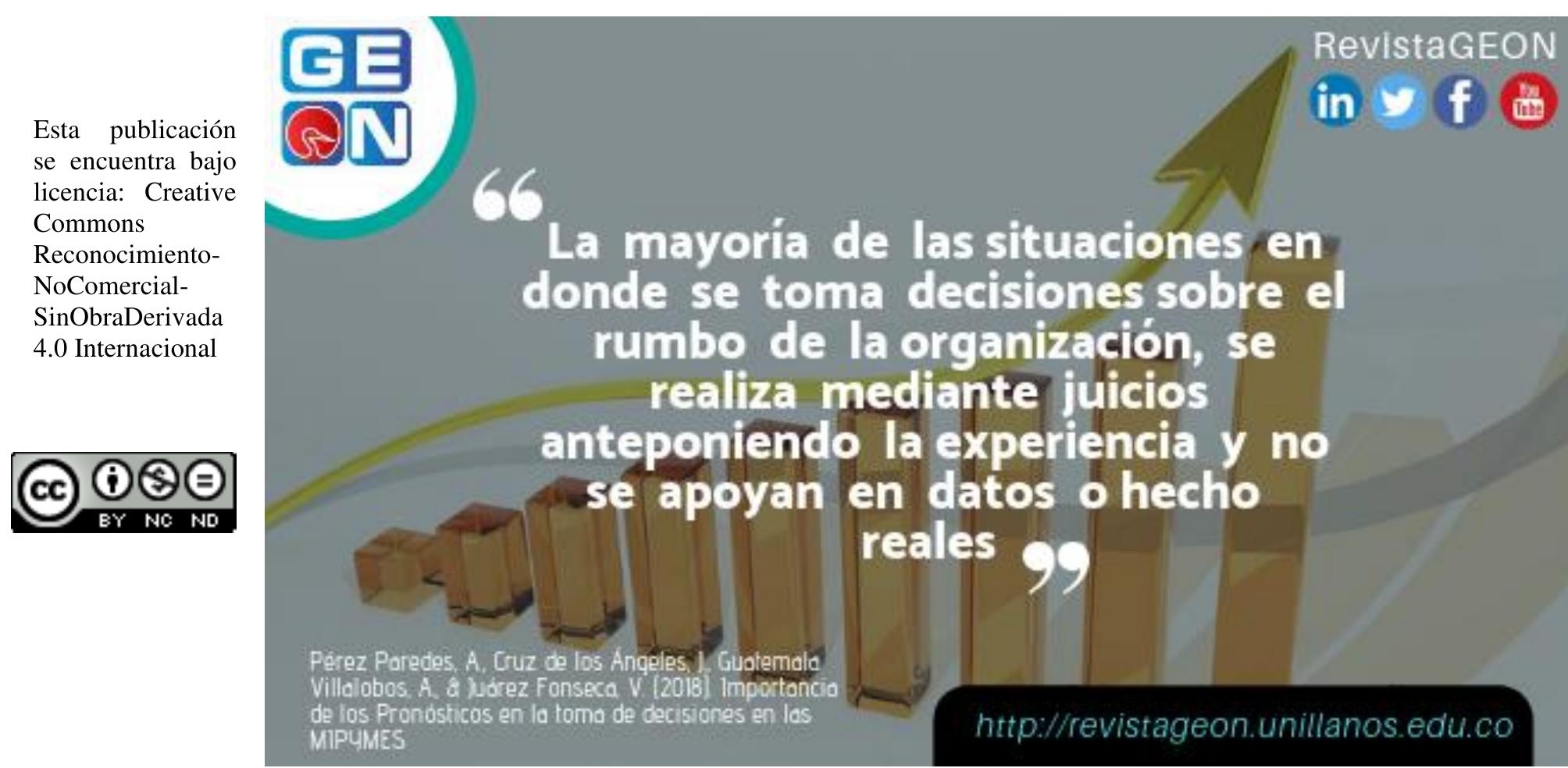


\section{REVISTA GEON}
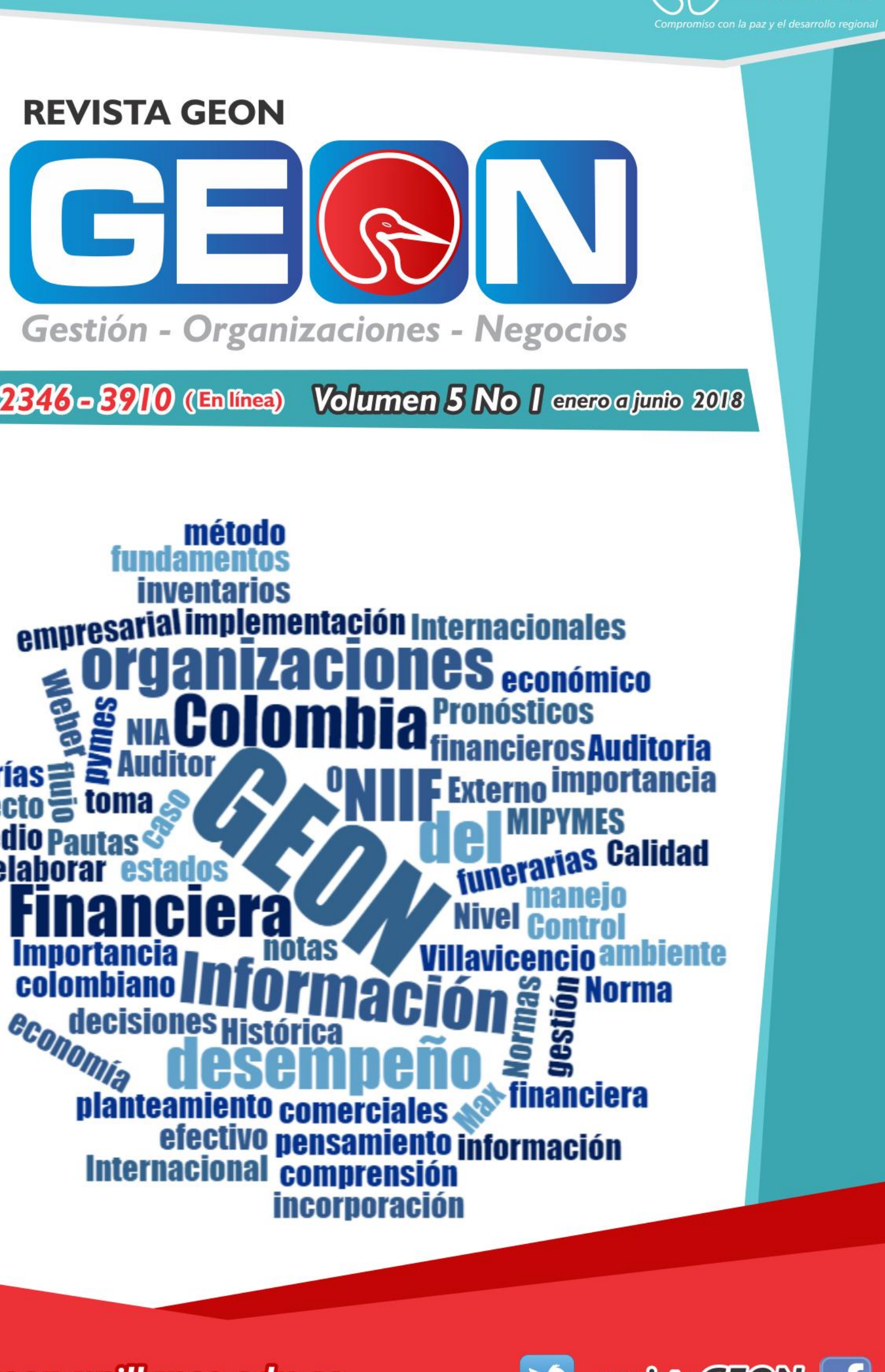

Gestión - Organizaciones - Negocios

ISSN 2346-3910 (Enlinea) Volumen 5No I enero a junio 2018

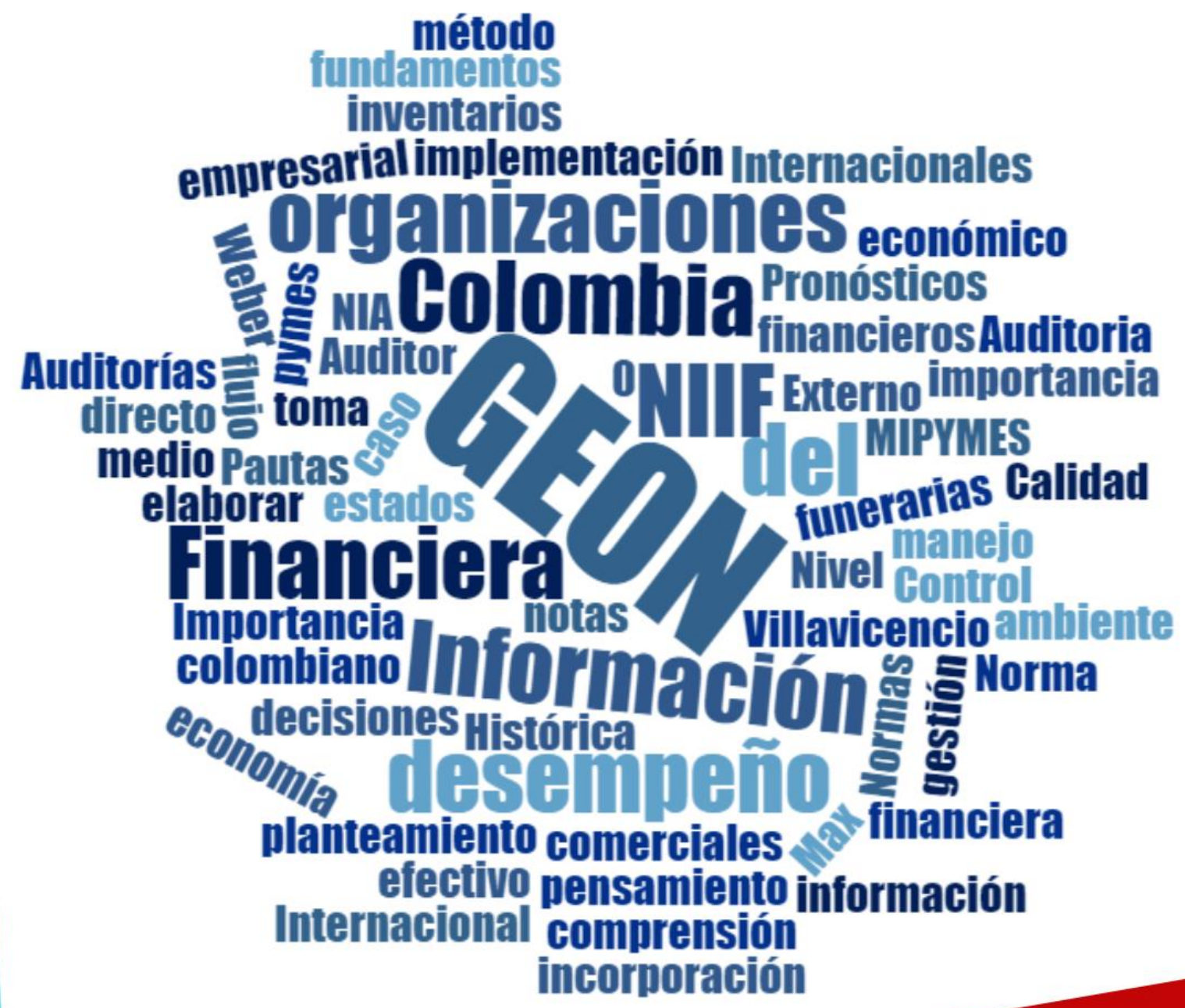

http://revistageon.unillanos.edu.co

revistaGEON f 


\section{Importancia de los Pronósticos en la toma de decisiones en las MIPYMES}

Importance of Forecasts in decision making in MSMEs

Alfredo Pérez Paredes, Doctor en Administración Pública - Magister en Administración, Cuerpo Académico Administración Aplicada - Benemérita Universidad Autónoma de Puebla BUAP, alfredoperez2001@ @otmail.com, México

Dr. Aurelio Cruz de los Ángeles, Doctor en Administración Pública - Magister en Administración, Cuerpo Académico Administración Aplicada - Benemérita Universidad Autónoma de Puebla BUAP pepejac52@ hotmail.com, México

Mtra. Araceli Ma. De Jesús Guatemala Villalobos, Magister en Administración de pequeñas y medianas empresas, Colaboradora Cuerpo Académico Administración Aplicada - Benemérita Universidad Autónoma de Puebla BUAP, araceli.guatemala@correo.buap.mx, México

Verónica Juárez Fonseca, Magister en Administración de pequeñas y medianas empresas, Colaboradora Cuerpo Académico Administración Aplicada - Benemérita Universidad Autónoma de Puebla BUAP, titosfonseca@yahoo.com.mx, México

\section{Resumen}

El estudio de los pronósticos como elementos en la toma de decisiones en las empresas tienen mayores áreas de oportunidad al interior de las organizaciones, esto se debe a que los pronóstico ayudan a los tomadores de decisiones a realizar juicios más precisos acerca de los eventos futuros y en esta parte las matemáticas resultan tener un papel importante, tal y como lo mencionan (Wilson \& Koerber, 1992) se ha demostrado que los métodos cuantitativos son útiles para hacer mejores pronósticos acerca del rumbo futuro de los eventos, por lo que se ha creado la necesidad que se desarrollen nuevos software que a través del uso de la computadora se generen más rápidamente estas predicciones, pero resulta fundamental comprender bien como se realizan los cálculos manualmente para utilizar posteriormente estas herramientas computacionales.

Para los fines de conocimiento que el administrador requiere saber sobre los diferentes métodos de pronósticos y con ello seleccionar el que cumpla con los objetivos internos, el autor Farrera Gutiérrez (2012) los divide en: Métodos Cualitativos entre los cuales se encuentran: Métodos 
Delphi, Analogía Histórica e Investigación de Mercados. Dentro de los Métodos Cuantitativos se encuentran: Promedio Móviles, Suavización Exponencial y Regresión Lineal. Es importante destacar que el conocer los diferentes métodos de pronósticos permite al tomador de decisiones al frente de una mipyme, acercarse lo más exacto a saber el comportamiento que tendrán los datos sobre los que se genera sus estimaciones a futuro.

En primer lugar, el presente artículo fundamenta los conceptos clave sobre los que se define un pronóstico y sus posibles aplicaciones dentro de las funciones principales en las que operan las empresas. Después se enuncia la clasificación general de los diferentes métodos para el cálculo de los pronósticos, señalando la utilidad de los métodos cuantitativos por ser los más precisos y cercanos a las estimaciones futura, así como la descripción sobre el significado de las series de tiempo y sus componentes, ya que se requiere conocer el comportamiento de los datos dentro de un periodo determinado.

De forma inmediata se explica las 5 técnicas aplicables a la medición del error en los pronósticos, explicando el significado de cada una así como el desarrollo a través de un ejemplo que permite reconocer la importancia de medir el error en los pronósticos que ayudará notablemente a seleccionar el método cercano a la estimación deseada. Finalmente, se describen los diferentes métodos cuantitativos, siendo los promedios móviles, suavización exponencial y regresión lineal que son los más utilizados para dicho cálculo, partiendo del comportamiento de datos históricos; lo anterior se desarrolla a base de un ejemplo con la finalidad de guiar al lector en su decisión al seleccionar uno u otro método.

Palabras clave. Pronósticos, Organizaciones, Toma de decisiones

\begin{abstract}
The study of forecasts as elements in decision making in companies have greater areas of opportunity within organizations, this is because the forecasts help decision makers to make more precise judgments about future events and In this part, mathematics turns out to have an important role, as mentioned (Wilson \& Koerber, 1992) it has been shown that quantitative methods are useful to make better predictions about the future course of events, so it has been created the need for new software to be developed through the use of the computer these predictions are generated more quickly, but it is fundamental to understand well how the calculations are made manually to later use these computational tools.
\end{abstract}


For the purposes of knowledge that the administrator requires to know about the different methods of forecasts and thus select the one that complies with the internal objectives, the author Farrera Gutiérrez (2012) divides them into: Qualitative Methods among which are: Delphi Methods, Historical Analogy and Market Research. Within the Quantitative Methods are: Moving Average, Exponential Smoothing and Linear Regression. It is important to note that knowing the different forecasting methods allows the decision maker in front of a MSME, to get as accurate as possible about the behavior of the data on which their future estimates are generated.

In the first place, the present article bases the key concepts on which a forecast is defined and its possible applications within the main functions in which the companies operate. The general classification of the different methods for the calculation of the forecasts is then stated, pointing out the usefulness of the quantitative methods as they are the most accurate and close to the future estimates, as well as the description of the meaning of the time series and their components, since it is required to know the behavior of the data within a certain period.

Immediately we explain the 5 techniques applicable to the measurement of the error in the forecasts, explaining the meaning of each one as well as the development through an example that allows to recognize the importance of measuring the error in the forecasts that will help notably to select the method close to the desired estimate. Finally, the different quantitative methods are described, being the moving averages, exponential smoothing and linear regression that are the most used for said calculation, starting from the behavior of historical data; The above is developed based on an example in order to guide the reader in his decision to select one or the other method.

Keywords. Forecasts, Organizations, Decision making

\section{Introducción}

En la actualidad, la toma de decisiones al interior de las micro, pequeñas y medianas empresas, representa un área de oportunidad porque dichas decisiones pueden ser la diferencia entre ser eficientes y competitivas, o, por el contrario, una mala decisión repercutiría hasta el punto de generar su cierre.
Esto se refleja cuando las nuevas condiciones económicas y globales que repercuten en México, no se toman en cuenta para determinar la factibilidad de apertura de las microempresas, aunado el éxito o fracaso de la mismas, por lo que para evitar el peligro al fracaso, es importante que se identifiquen los factores de éxito y la toma de decisiones que le 
permita estructurar estrategias para su permanencia, crecimiento y desarrollo.

De conformidad con los datos estandarizados por parte de la autora Pierina (2018) muestra que durante el primer año de arranque mueren el $50 \%$ de las pymes; para el segundo año suman otro $25 \%$ y para el quinto año muere otro $15 \%$, dejándonos sólo un $10 \%$ del total que sobrevivirá madurando como pequeña empresa; lo anterior como resultado probablemente por una toma de decisiones de forma empírica, es decir, no se realiza de manera sistematizada y esto provoca que cuando se decide cuanto producir, cuanto comprar, cuanto vender, cuantos impuestos pagar, cuántos empleados extras contratar $y$ toda una serie de decisiones que requieren pronosticar, se realice en función de presentimientos o suposiciones que no cuentan con ningún fundamento teórico o técnico.

Sin duda alguna esto representa un problema porque no existe ningún método exacto o preciso para adivinar qué sucederá en el futuro, sobre todo en los entornos tan cambiantes en donde las condiciones económicas, políticas $\mathrm{y}$ sociales influyen directamente en las empresas, esto genera aún más la complicación de cómo decidir las acciones del futuro que les permita optimizar los recursos de la empresa, para alcanzar los objetivos organizaciones previamente establecidos.

Por ello el tema de los pronósticos resulta ser de gran interés para predecir de una manera sistemática en la toma de decisiones que tienen que ver con situaciones que se presentan en el quehacer diario de todas las áreas funcionales de las organizaciones y que si no se realiza correctamente pone en riesgo la utilización inadecuada de los recursos que no siempre son excedentes, en la mayoría de los casos sobre todo en las micros y pequeñas empresas, resultan ser escasos.

Con estos fundamentos el presente artículo pretende demostrar la importancia que tiene este tema para la toma de decisiones al interior de las organizaciones, de esta forma contribuirá al interesado en el tema de pronósticos, dando a conocer los distintos modelos que le ayudarán a predecir de una manera sistemática las decisiones sobre las que se apoyan las actividades operativas cotidianas y con ello generar estrategias que le permitan mantener y madurar el desarrollo interno de la MIPYME.

El presente artículo se desarrolla presentando en un primer apartado su definición, posteriormente cuáles son sus orígenes a partir de las series de tiempo, la determinación del error en los pronósticos, los tipos de pronósticos que se pueden calcular y por último se presentaran algunos ejemplos de cada uno de ellos, que les permita al lector comprender como se calculan $y$ en su caso aplicarlos a situaciones específicas que se presentan al interior de las organizaciones.

\section{Desarrollo}

En esta parte del trabajo se hablará acerca de los pronósticos, así como la clasificación de los métodos existentes para calcular los pronósticos $\mathrm{y}$ 
posteriormente se presentarán como utilizar dichos métodos sobre todo los que se proponen para la toma de decisiones que son los métodos cuantitativos.

\section{Pronósticos}

El pronóstico ayuda a los tomadores de decisiones a realizar juicios más precisos acerca de los eventos futuros que podrían repercutir en las operaciones internas, por lo que en esta parte las matemáticas resultan tener un papel importante, tal y como lo mencionan Wilson \& Koerber (1992) ya que en su investigación demostraron que los métodos cuantitativos son útiles para hacer mejores pronósticos acerca del rumbo futuro de los eventos, por lo que se ha vuelto necesario que se desarrollen nuevos software que a través del uso de la computadora genera más rápidamente estas predicciones, pero resulta fundamental comprender bien como se realizan los cálculos manualmente para utilizar posteriormente estas herramientas computacionales.

Una primera definición indica que un pronóstico es la estimación del valor futuro de una variable mediante la aplicación de métodos y procedimientos que contribuyan a reducir el margen de error, haciendo uso además del buen juicio y experiencia del responsable de dicha estimación (Farrera Gutierrez, 2012).

Otra definición la menciona el autor Mendoza Manuel (2010) la cual describe que un pronóstico es una afirmación sobre un evento cuya ocurrencia no es segura.
La definición propia que se propone para mejor entendimiento de esta investigación del término pronósticos es la siguiente:

Proceso de calcular una situación futura, a través de métodos cualitativos o cuantitativos, a partir de información histórica que permita una toma de decisiones lo más acertada posible.

Como se ha mencionado, el pronóstico representa una estimación del futuro partiendo de datos históricos $\mathrm{y}$ considerando la experiencia que tienen tanto el tomador de decisiones como el que determina el pronóstico.

Algunas de las aplicaciones que se le pueden dar a los pronósticos son las que menciona Farrera (2012) siendo las siguientes:

Mercadotecnia. - Es probable que el uso más común de los pronósticos en los negocios sea la estimación de la demanda para planear las estrategias de ventas, además de la participación del mercado y el posicionamiento de una marca, entre otras.

Producción. - Es necesario hacer estimaciones de las variables operativas de una empresa, tales como: productividad, mermas, niveles de inventarios, defectos de producción, cantidad de materias primas, entre otros.

Finanzas. - todas las variables que tienen que ver con las finanzas de una empresa necesitan estimarse también, entre ellas: costos y gastos, rotación de activos y pasivos, tasas de interés, tasas financieras y utilidades. 
Recursos Humanos. - Sin duda el factor humano es el que mueve a las organizaciones, y no menos importante es establecer estimaciones sobre los niveles de ausentismo, accidentes de trabajo, rotación de personal, enfermedades e índices de desempeño.

Como menciona dicho autor, estas son algunas de las aplicaciones que se les pueden dar a los pronósticos al interior de cualquier organización productiva y puede haber muchas más, sin embargo, esto depende claramente de las características y condiciones que tienen en particular cada una de las empresas.

\section{Clasificación de los Métodos para calcular Pronósticos}

Con la finalidad de hacer frente a la incertidumbre sobre las decisiones que se toman al interior de las organizaciones, es necesario conocer los diferentes métodos de pronósticos a fin de respaldar la elaboración correcta de los mismos, haciendo a un lado la intuición en que regularmente se apoyan las decisiones, y con ello justificar los resultados en base a un costo-beneficio que desee la empresa; se debe considerar que situación específica se debe atender para determinar que método es el más conveniente; por ello, a continuación se presenta como se clasifican:

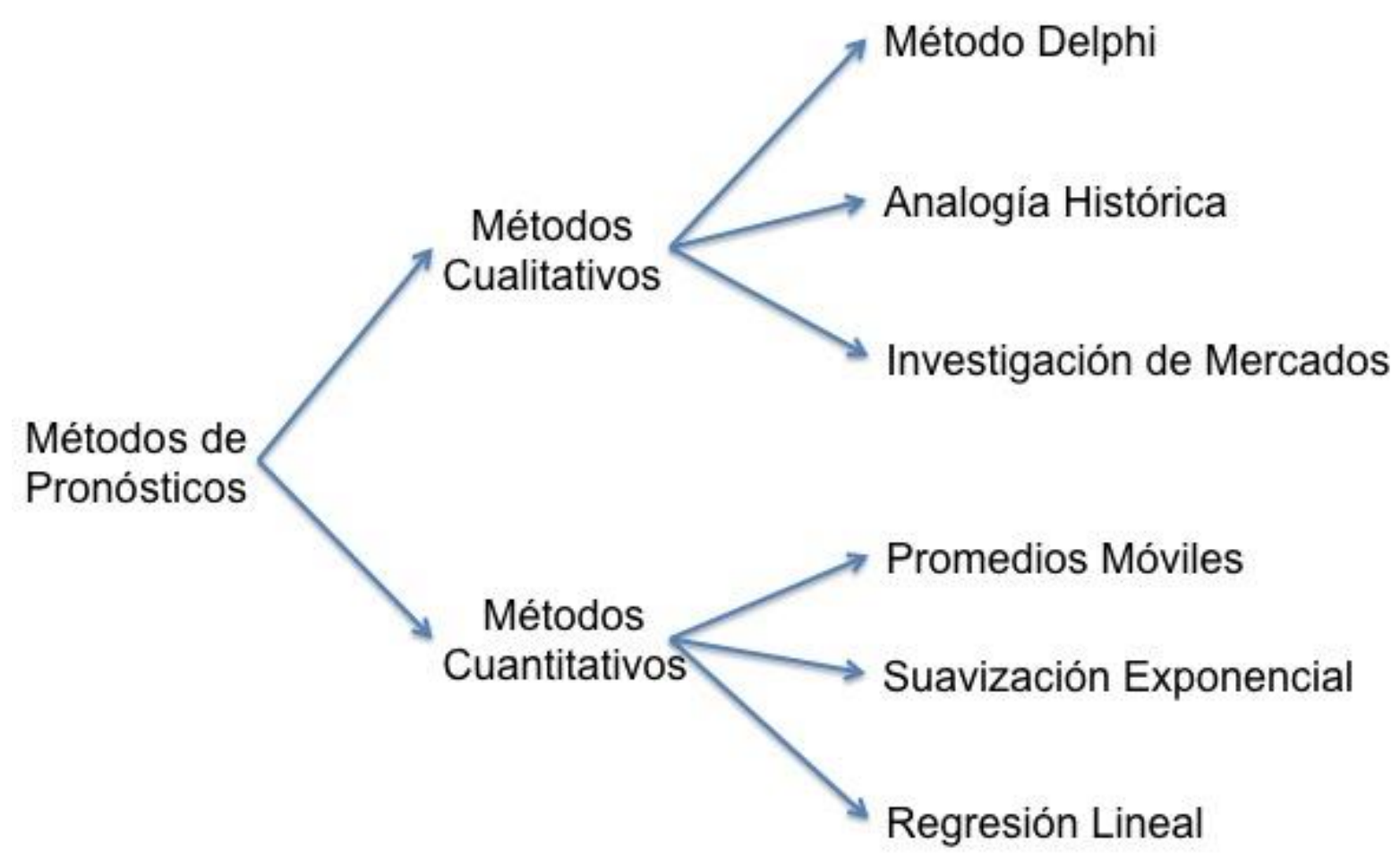

Figura No. 1 Clasificación de Pronósticos Fuente: Elaboración Propia 
Métodos cualitativos.- Según Gallagher (2003) con frecuencia se usan para situaciones a largo plazo, altamente inciertas en las cuales el empleo de un modelo matemático no parece apropiado. En estos casos, es normal que el juicio subjetivo sea la base para llegar a un pronóstico y entre ellas se encuentran las siguientes:

Método Delphi. - Landeta (2002) lo define como el método de estructuración de un proceso de comunicación grupal que es efectivo a la hora de permitir a un grupo de individuos, como un todo, tratar un problema complejo.

Analogía Histórica. - Modelo cualitativo que consiste en identificar el producto o servicio que se pronostica con alguno similar al cual se le conoce su historia pasada. Este modelo es importante en la planificación de nuevos productos en donde un pronóstico puede ser derivado de la historia de un producto similar.

Investigación de Mercados. - Es una técnica que permite recopilar datos, de cualquier aspecto que se desee conocer para, posteriormente, interpretarlos y hacer uso de ellos. Sirven al comerciante o empresario para realizar una adecuada toma de decisiones y para lograr la satisfacción de sus clientes.

Métodos cuantitativos. - Aunque varía su complejidad, todos emplean modelos matemáticos que requieren datos para las variables independientes con objeto de generar un pronóstico, entre los que se encuentran los siguientes:

Promedios Móviles. - Consiste en calcular un valor promedio en base a los datos históricos que se dispongan y utilizar este valor como pronóstico para un periodo en el futuro. Para eliminar la aleatoriedad de los datos obtenidos hay que considerar el promedio de los últimos valores observados y usarlo como pronóstico para un periodo próximo. El número de observaciones que se habrán de utilizar en este promedio se especifica de antemano y permanece constante.

Suavizamiento Exponencial. - Se puede considerar como una evolución del método de Promedios Móviles, que considera el promedio de una serie de tiempo con factor de autocorrección que busca ajustar los pronósticos en dirección contraria a las desviaciones del pasado que se ve modificada por un coeficiente de suavización. Para la realización de este pronóstico se deben considerar el pronóstico anterior, el dato histórico anterior y el coeficiente de suavización denominado alfa y que debe ser menor $1 \mathrm{y}$ mayor a 0 .

Regresión Lineal. - También denominada de mínimos cuadrados se puede utilizar para determinar pronósticos de series de tiempo, en particular cuando la variable dependiente cambia como resultado del transcurso del tiempo y por ello se requiere utilizar la información histórica de la variable que se desea pronosticar. $Y$ esto se consigue utilizando una técnica estadística usada para resolver una ecuación matemática que muestra cómo se relacionan las variables.

Como se ha descrito, existen diversos métodos para calcular los pronósticos que dependiendo de lo que se desea predecir con las variables, se utiliza un método determinado. En este punto, es 
importante destacar que en este artículo se presentarán los métodos cuantitativos ya que se basan en información o datos históricos o de hechos reales y no en juicios basados en la intuición (por la falta de datos), permitiendo al administrador centrar la toma de decisiones que repercutirá de manera efectiva al ser aplicada en las operaciones de su empresa.

\section{Las Series de Tiempo y sus Componentes}

De acuerdo al sector en el que se desenvuelven las pymes (industrial, comercial o servicios) éstas desean conocer el comportamiento futuro de ciertos fenómenos que les ayude a planear y prevenir, es decir, poder predecir lo que ocurrirá con una variable en el futuro partiendo de hechos pasados por lo que hacen referencia a la presentación de una serie de datos históricos que se registran para su observación y análisis, considerando que siempre se capturan en un periodo de tiempo regular que puede ser anual, semestral, mensual o inclusive diario, dependiendo de la variable que se esté revisando; dicho comportamiento de los datos se le conoce como serie de tiempo, que no es más que la sucesión periódica de datos históricos (Farrera Gutierrez, 2012).

Lo anterior permite en un primer momento, determinar si se presentan ciertos patrones o pautas no aleatorias, conforme transcurren en el tiempo; también permite aislar y estudiar sus componentes a fin de proporcionar claves para predecir futuros cambios y esto hace posible adicionalmente pronosticar las acciones futuras en cada una de las áreas funcionales de la organización así como otros aspectos que estén sincronizados.

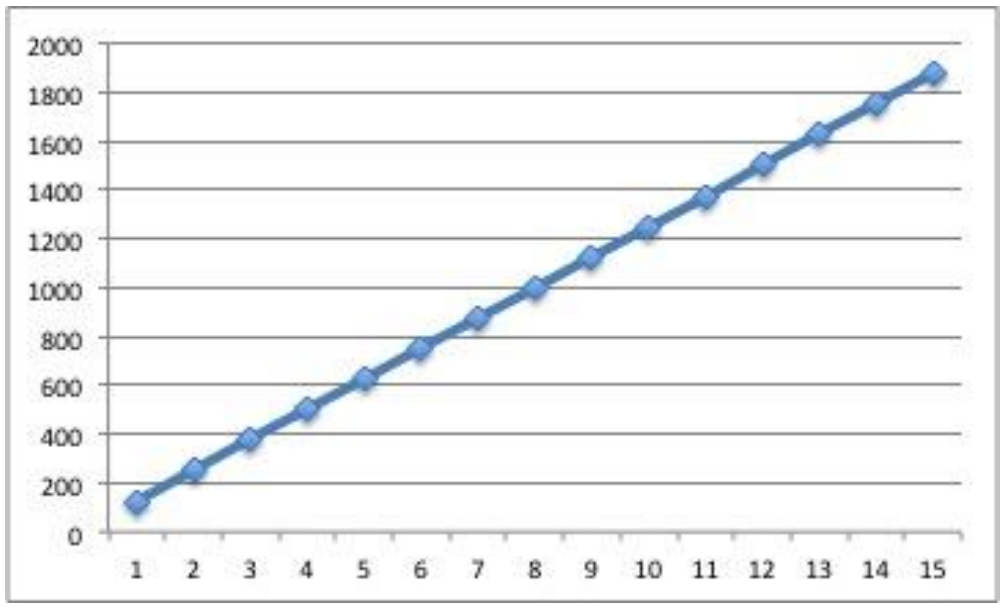

Gráfica No. 1 Componente de Tendencia

Fuente: Elaboración propia

Las series de tiempo tienen principalmente cuatro componentes que son el de Tendencia, Fluctuaciones Cíclicas, Variaciones estacionales y Variaciones Irregulares, Wilson (1992) los describe a continuación. La tendencia en una serie de tiempo es el cambio del nivel de los datos a largo plazo. Si para un periodo prolongado la serie se mueve hacia arriba, se dice que los datos muestran una tendencia positiva. Si el nivel de los datos disminuye con el tiempo, se dice que existe una tendencia negativa. Los datos se consideran estacionario cuando no hay una tendencia positiva ni negativa, es decir, la serie es en esencia plana a largo plazo.

El componente de Fluctuaciones Cíclicas se representa mediante movimientos de los datos hacia arriba y hacia abajo -parecidos a una ondaalrededor de la tendencia a largo plazo. Las fluctuaciones cíclicas son de mayor duración y son menos regulares que las 
fluctuaciones estacionales. Las causas de las fluctuaciones cíclicas son también menos evidentes. Usualmente se atribuyen a los ascensos y descensos del nivel general de la actividad de los negocios que con frecuencia se denominan ciclos de negocios.

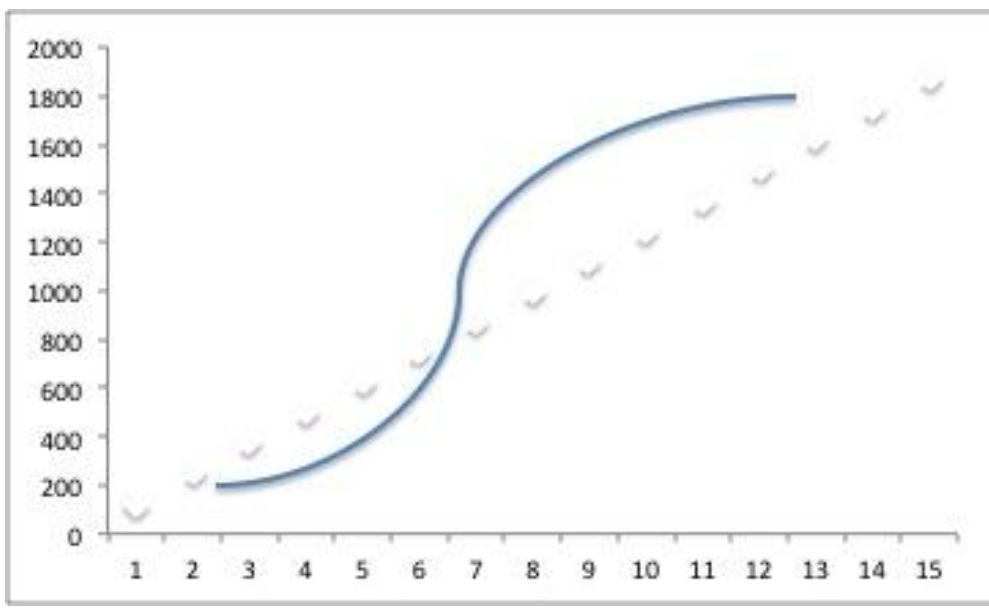

Grafica No. 2 Componente de Fluctuaciones Cíclicas Fuente: Elaboración Propia

Componente de Variaciones Estacionales que en una serie de tiempo cuando existe una variación regular del nivel de los datos que se repite cada año para el mismo período. Por ejemplo, los productos o servicios que tienen tasas de ocupación muy alta en los meses de diciembre. Las ventas al menudeo para muchos productos tienden a presentar un pico en noviembre y en diciembre debido a las ventas de vacaciones y esto representa patrones estacionales.

Componente Irregular de una serie de tiempo se representa porque contiene las fluctuaciones que no son parte de los otros tres componentes. Se le suele denominar fluctuaciones aleatorias y como tales, son las más difíciles de capturar en un modelo de pronóstico.

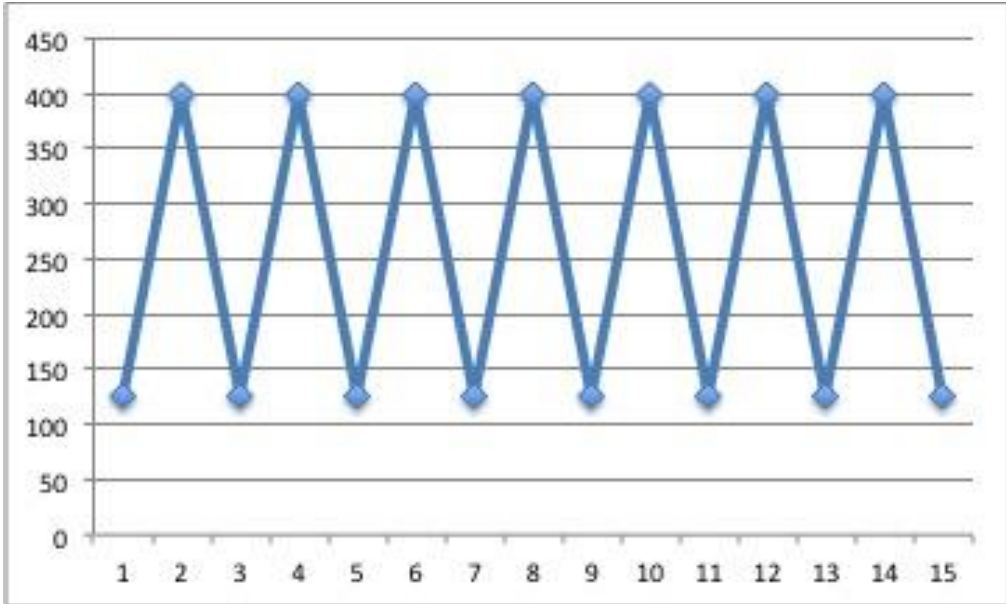

Gráfica No. 3 Componente de Variaciones Estacionales Fuente: Elaboración Propia

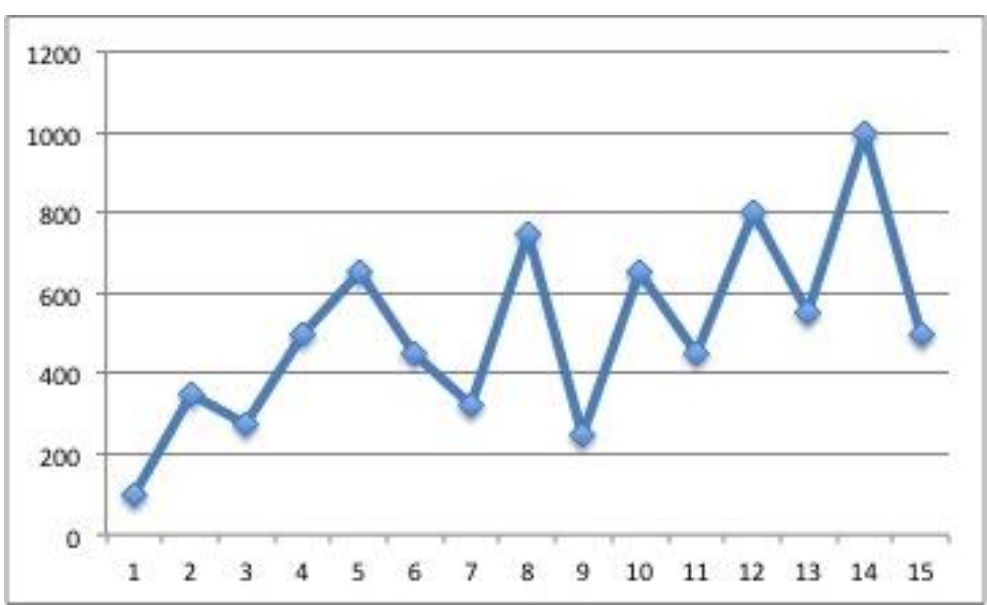

Gráfica No. 4 Componente Irregular

Fuente: Elaboración propia

Como se observa, el comportamiento de los datos históricos es un factor determinante para seleccionar el método de pronóstico más adecuado para determinarlo, por ello la importancia de entender los componentes de las series de tiempo.

Técnicas en la Medición del Error en los Pronósticos

Al realizar un pronóstico lleva implícito un margen de error, dicho margen de error puede ser grande o 
pequeño, lo que nos llevaría a determinar el grado de precisión o exactitud de la estimación, por lo que se vuelve necesario aplicar diferentes mediciones del error a través de técnicas de índole estadístico para determinar qué tan útiles son las estimaciones determinadas o si será necesario aplicar otro tipo de métodos para llegar a la mayor precisión del pronóstico.

Farrera Gutiérrez (2012) define al error como la desviación o variación que existe entre el valor real de la variable y su valor pronosticado, por lo que las siguientes técnicas son las que con mayor frecuencia se aplican ya que se enfocan a una " $n$ " serie de tiempo, con ello ayudan a determinar qué tan exacto es el pronóstico de la demanda, es decir, si el pronóstico se encuentra sub estimado o sobre estimado, de ésta manera permite identificar un modelo de pronóstico que arroje el menor error posible; lo anterior pone en comparación los datos de la demanda estimada, con los datos de cada una de las técnicas en cuestión.

Por lo tanto, una vez que se ha determinado el cálculo se deberá evaluar los pronósticos obtenidos a través de algunas medidas del error que indicarán cual es el mejor pronóstico; esto se logra identificar cuando estas medidas son cercas a cero, sin importar si esta cercanía se presenta con valores negativos $\mathrm{o}$ positivos, es decir, si se cuenta con los resultados de dos pronósticos obtenidos con los mismos datos, se determinará que el pronóstico más adecuado es aquel en que las medidas del error estén más cercanas al cero.
La presente explicación toma como referencia un ejemplo que ilustra y orienta al tomador de decisiones a aplicar cada una de las técnicas con los datos de su demanda real y la pronosticada.

Las cinco técnicas más utilizadas dentro de la medición del error se aplican a mediciones de pronósticos de series de tiempo, como se ha explicado en el subtema anterior, ya que con mayor frecuencia las estimaciones se realizan sobre el comportamiento de datos históricos y dichas mediciones se aplican a los pronósticos de series de tiempo, permitiendo al administrador al frente de una pyme, acercarse con exactitud a aquel modelo que le permita lograr los objetivos organizacionales.

Las técnicas son las siguientes:

Error Medio (ME). - Esta medida permite determinar qué tan cercanos o alejados están los pronósticos de los datos originales.

Formula: $M E=\frac{\sum e}{n}$

Donde: $\quad \mathrm{e}=$ error

$$
\mathrm{n}=\text { número de errores }
$$

Desviación Media Absoluta (DMA).Esta medida considera el error en valor absoluto, y elimina la cancelación de valores que se da en la medida anterior, debido a que se eliminan los valores negativos. Esta suele ser la medida preferida para medir el error el pronóstico.

Formula: $D M A=\frac{\Sigma e}{n}$ 
Error Cuadrático Medio (ECM).Esta medida es similar a la varianza de una muestra aleatoria, debido a que se determina el error elevado al cuadrado.

Formula: $E C M=\frac{\Sigma e^{2}}{n}$

Error Porcentual Medio Absoluto (EPMA).- Esta medida elimina la ambigüedad de las unidades, ya que depende del resultado que se obtiene de dividir el error entre el dato original considerando su valor absoluto, se expresa en porcentaje.

Formula:

$$
E P M A=\frac{\Sigma \mathrm{e} / \mathrm{d}}{n}
$$

Desviación Estándar del Error $\left(\sigma^{2}\right)$.A partir de esta se considera si los errores están normalmente distribuidos y por ello representa una aproximación de la desviación Estándar.

Formula: $\sigma^{2}=1.25 * D M A$

A continuación, se presenta un ejemplo de cómo calcular estas medidas del error y como determinar el que más conviene.

Donde: $d=$ dato original

Tabla No. 1 Determinación del Error

\begin{tabular}{|c|c|c|c|c|c|c|c|c|c|c|c|}
\hline Periodo & $\begin{array}{l}\text { Dato } \\
\text { (d) }\end{array}$ & Pronóstico 1 & Pronóstico 2 & e 1 & e 2 & $|\mathrm{e}| 1$ & $|\mathrm{e}| 2$ & $e^{2} 1$ & $e^{2} 2$ & $|\mathrm{e} / \mathrm{d}| 1$ & $|e / d| 2$ \\
\hline 1 & 210 & 200 & 210 & -10 & 0 & 10 & 0 & 100 & 0 & 0.05 & 0 \\
\hline 2 & 280 & 260 & 220 & -20 & -60 & 20 & 60 & 400 & 3600 & 0.08 & 0.27 \\
\hline 3 & 220 & 190 & 170 & -30 & -50 & 30 & 50 & 900 & 2500 & 0.16 & 0.29 \\
\hline 4 & 310 & 320 & 280 & 10 & -30 & 10 & 30 & 100 & 900 & 0.03 & 0.11 \\
\hline 5 & 260 & 240 & 260 & -20 & 0 & 20 & 0 & 400 & 0 & 0.08 & - \\
\hline 6 & 350 & 390 & 400 & 40 & 50 & 40 & 50 & 1600 & 2500 & 0.10 & 0.13 \\
\hline 7 & 280 & 260 & 270 & -20 & -10 & 20 & 10 & 400 & 100 & 0.08 & 0.04 \\
\hline 8 & 210 & 210 & 220 & 0 & 10 & 0 & 10 & 0 & 100 & - & 0.05 \\
\hline Total & & & & -50 & -90 & 150 & 210 & 3900 & 9700 & 0.578888 & 0.88148 \\
\hline
\end{tabular}

Fuente: Elaboración propia

En este ejemplo con los datos "d" se determinaron dos pronósticos el 1 y 2 que para este ejemplo se encuentran representadas en las primeras cuatro columnas de la tabla No. 1 y a partir de la columna cinco se inician los cálculos para determinar cuál de los dos es el más acertado y para ello se inicia el cálculo de las cinco medidas del error.

Procedimiento:

Paso 1. Se determina el valor del error que se representa con la letra "e" (columna 5 y 6) y se calcula restando al pronóstico el dato original, en estos 
cálculos se pueden obtener valores positivos o negativos.

Paso 2. Se determina el error medio (EM) en donde se suman todos los errores del pronóstico 1 que para este caso es de 50 y para el pronóstico 2 que para este caso es de -90 (Columna 5 y 6) y en ambos casos se divide entre 8 que representan el número de errores que se han determinado. El error medio es para el pronóstico 1 es de -6.25 y para el pronóstico 2 es de 11.25 , por lo tanto, el pronóstico más acertado es el primero porque la medición es la más cercana al valor de cero.

Paso 3. Se determina la desviación media absoluta (DMA) en donde se suman todos los valores absolutos de los errores (Columna 7 y 8), con el propósito de evitar la cancelación de los valores negativos de los errores. Para este ejemplo la suma de los valores absolutos del error para el pronóstico 1 fue de 150 que dividiéndolo entre los 8 errores resultó 18.75 y para el pronóstico 2 la suma fue de 210 que dividiéndolo entre los ocho errores resultó 26.25 .

Paso 4. Se determina el Error Cuadrático Medio (ECM) en donde el valor de los errores se eleva al cuadrado (Columnas 9 y 10) y posteriormente se suman. Para este ejemplo se obtuvieron 3,900 para el pronóstico 1 que al dividirlo entre los ocho errores el resultado fue 487.5 y 9,700 para el pronóstico 2 que al dividirlo entre los ocho errores generó un resultado de 1,212.5

Paso 5. Se determina el Error Porcentual Medio Absoluto (EPMA) en donde se determina el valor absoluto de la división entre el error generado entre cada uno de los datos históricos (Columna 11 y 12) y se suman, para el caso del primer pronostico se obtuvo una sumatoria para el pronóstico 1 de .5788 que al dividirlo entre los ocho errores generó un resultado de .0723 o $7.23 \%$ y para el pronóstico 2 se generó una sumatoria de .8814 que al dividirlo entre los ocho errores se obtuvo .1102 o $11.02 \%$.

Paso 6. Se calcula la Desviación Estándar del Error $\left(\sigma^{2}\right)$ en donde lo único se realiza es multiplicar 1.25 por la DMA que para este ejercicio fue de 18.75 para el pronóstico 1 y 26.25 para el pronóstico 2 . Se realizaron las multiplicaciones y los resultados fueron 23.44 y 32.81 de desviación Estándar del Error para cada pronóstico.

Como se observa para este caso el pronóstico valido para este ejercicio es el número 1 , debido a que en las medidas del error que se calcularon, fueron menores y más cercanas al cero sin importar si son valores positivos $\mathrm{o}$ negativos. Cabe destacar que esta situación no siempre es así, debido a que en algunas ocasiones los valores de algunas medidas no reflejan la misma tendencia hacia un resultado, pero en la mayoría de las medidas permitirá identificar cual es el mejor pronóstico.

\section{Métodos Cuantitativos De Los Pronósticos}

En este apartado se presentan tres ejemplos para el cálculo de pronósticos que son el de promedios móviles, suavización exponencial y regresión lineal. 
Promedios Móviles. Se inicia presentando un ejemplo del método más sencillo para determinar los pronósticos, a continuación se presenta un ejemplo.

La primera acción es verificar que tipo de componente tienen las ventas registradas y para ello se grafican las ventas para ver cuál es el comportamiento que tienen y se obtuvo la siguiente gráfica.

Como se muestra en la gráfica número cinco los datos tienen un comportamiento ascendente y descendente lo que refleja que se está ante un componente estacional, por lo que resulta apropiado aplicar el método de pronósticos móviles. No hay que perder de vista la pertinencia de ocupar este método cuando se trata de componentes de variación estacional o cíclica.

Tabla No. 2 Ejemplo de Promedios Móviles

Fuente: Elaboración Propia

\begin{tabular}{|l|r|l|l|}
\hline Mes & Ventas & Promedio Móvil 2 & Promedio Móvil 3 \\
\hline 1 & $7,100.00$ & & \\
\hline 2 & $6,953.00$ & & \\
\hline 3 & $7,268.00$ & $7,026.50$ & \\
\hline 4 & $7,023.00$ & $7,110.50$ & $7,107.00$ \\
\hline 5 & $7,555.00$ & $7,145.50$ & $7,081.33$ \\
\hline 6 & $7,021.00$ & $7,289.00$ & $7,282.00$ \\
\hline 7 & $7,558.00$ & $7,288.00$ & $7,199.67$ \\
\hline 8 & $7,245.00$ & $7,289.50$ & $7,378.00$ \\
\hline 9 & $7,600.00$ & $7,401.50$ & $7,274.67$ \\
\hline 10 & $7,300.00$ & $7,422.50$ & $7,467.67$ \\
\hline 11 & $7,500.00$ & $7,450.00$ & $7,381.67$ \\
\hline 12 & $7,000.00$ & $7,400.00$ & $7,466.67$ \\
\hline 13 & Pronóstico & $7,250.00$ & $7,266.67$ \\
\hline
\end{tabular}

Grafica No. 5 Comportamiento de los datos de la Tabla No. 2

Fuente: Elaboración propia

En la Tabla no. 2 se presenta en la columna 1 el periodo de tiempo que para este caso es un mes pero pueden ser cualquier otro periodo de tiempo; en la columna 2 se presentan las ventas realizadas cada mes; en la columna número 3 se presentan los resultados del pronóstico para el mes 13 utilizando un promedio móvil con 2 datos de tal forma 
que se promedió el valor de 7,100 y el de 6,953 dando un resultado de $7,026.50$ que se convierte en el pronóstico del mes 3, posteriormente se promedian los valores de 6,953 y 7,268 generando un promedio de $7,110.50$ que se convierte en el pronóstico del mes número 4 y así se realizan sucesivamente hasta llegar al pronóstico del mes número 13 que fue de $7,250.00$ al promediar los meses 11 y 12; por último en la columna 4 también se presentan los resultados del pronóstico para el mes 13 utilizando un promedio móvil con 3 datos de tal forma que se promedió el valor de 7,100, 6,953 y 7,268 dando un resultado de 7,107 que se convierte en el pronóstico del mes 4, posteriormente se promedian los valores de 6,953, 7,268 y 7,023 generando un promedio de $7,081.33$ que se convierte en el pronóstico del mes número 5 y así se realizan sucesivamente hasta llegar al pronóstico del mes número 13 que fue de $7,266.67$ al promediar los meses 10, $11 \mathrm{y}$ 12 , este método es muy sencillo de realizar porque solamente se considera como pronóstico, cada uno de los promedios que se van obteniendo, por tal motivo, se considera que los resultados pueden ser poco precisos, toda vez que no se pondera en ningún momento todos los datos y esto le resta cierta validez o confiabilidad.

Sin embargo, este tipo de método puede resultar confiable, para algún tipo de empresas, que por su tamaño, giro o situaciones específicas en el mercado le resulte lo adecuado para pronosticar, aquí realmente lo importante es definir los periodos de tiempo, recabar de manera adecuada los datos y seleccionar algún método que más se apegue a la realidad de cada organización. Para este ejercicio que se presenta se obtuvieron dos pronósticos para el mes 13. ¿Cuál es el más acertado? La respuesta es aquel que a la hora de calcular las medidas del error se aproxime más al cero.

\section{Suavización Exponencial. Dentro} de los métodos cuantitativos, la suavización exponencial se identifica utilizando dentro de la fórmula, un promedio ponderado de valores de series de tiempo pasadas como pronóstico; su utilidad se da al proporcionar un alto nivel de precisión para pronósticos de corto alcance, es decir, para un pronóstico del siguiente periodo (Fernanda, 2016), se describe mediante el siguiente ejemplo.

Para este método se considera un factor de suavización denominado alfa $(\alpha)$ que debe adquirir un valor menor de $1 \mathrm{y}$ mayor a 0 es decir puede ser un valor de $0.05,0.10$ o inclusive hasta 0.90 . Este valor se coloca de acuerdo con el criterio de quien calcula el pronóstico, sin embargo, en el momento de calcular las medidas del error, se pueden realizar ajustes en el factor de suavización hasta que se encuentre el más apropiado para la organización.

La fórmula para determinar el pronóstico es la siguiente:

$$
\mathrm{P}=\alpha * \text { Dato anterior }+((1-\alpha) *
$$
Pronóstico anterior)

También es importante aclarar que al igual que los promedios móviles este método también se utiliza para datos que tienen un componente cíclico y de estacionalidad, por ello para el presente ejemplo se utilizaran los mismos datos para determinar los pronósticos. 
En la tabla número 3 se presenta en la columna 1 y 2 los datos relacionados con el mes y las ventas respectivamente y en la columna 3 se inician los cálculos del pronóstico con un $\alpha$ igual a 0.10 .

Para utilizar la formula presentada anteriormente se debe considerar que en el mes uno no puede haber pronóstico porque la formula pide el pronóstico anterior y este no existe, por lo tanto, el pronóstico del mes uno, tiene que ser igual al dato del mes uno, es decir, tendremos que colocar 7,100 . Otros autores para colocar el valor de este pronóstico inicial determinan el promedio de todos los datos y es el que se coloca, para este caso se utilizará el mismo dato del periodo inicial.

Tabla No. 3 Ejemplo de suavización Exponencial

\begin{tabular}{|l|l|l|l|}
\hline Mes & Ventas & $\begin{array}{l}\text { Suavización } \\
\alpha=0.10\end{array}$ & $\begin{array}{l}\text { Suavización } \\
\alpha=0.20\end{array}$ \\
\hline 1 & $7,100.00$ & $7,100.00$ & $7,100.00$ \\
\hline 2 & $6,953.00$ & $7,100.00$ & $7,100.00$ \\
\hline 3 & $7,268.00$ & $7,085.30$ & $7,070.60$ \\
\hline 4 & $7,023.00$ & $7,103.57$ & $7,110.08$ \\
\hline 5 & $7,555.00$ & $7,095.51$ & $7,092.66$ \\
\hline 6 & $7,021.00$ & $7,141.46$ & $7,185.13$ \\
\hline 7 & $7,558.00$ & $7,129.42$ & $7,152.30$ \\
\hline 8 & $7,245.00$ & $7,172.27$ & $7,233.44$ \\
\hline 9 & $7,600.00$ & $7,179.55$ & $7,235.76$ \\
\hline 10 & $7,300.00$ & $7,221.59$ & $7,308.60$ \\
\hline 11 & $7,500.00$ & $7,229.43$ & $7,306.88$ \\
\hline 12 & $7,000.00$ & $7,256.49$ & $7,345.51$ \\
\hline 13 & Pronóstico & $7,230.84$ & $7,276.41$ \\
\hline & Fuente: Elaboración propia \\
\hline
\end{tabular}

Posteriormente se aplica la fórmula para determinar el pronóstico del mes número 2 y se encontró que al multiplicar $0.10 * 7,100$ y sumarle la multiplicación de 0.90 (que se obtiene de $1-0.10$ ) * 7,100 , el pronóstico generado también es 7,100 , esto provocado al tener en ambos extremos de la ecuación la misma cantidad.

Resultado diferente, es que el que se obtiene para el mes número 3 en donde se multiplica $0.10 * 6,953$ y se le suma la multiplicación de $0.90 * 7,100$ el resultado del pronóstico es de 7,070.60, esto debido a que el valor del dato número 2 ya se modificó.

Este procedimiento se repite hasta llegar al pronóstico del mes 13 en donde se multiplico $0.10 * 7,000$ y se le sumó la multiplicación de $0.90 * 7,256.49$ y entonces se determina un pronóstico final de 7,230.84 con un factor de suavización del 0.10 .

En la columna 4 se realiza el mismo procedimiento y solo se modifica el valor de $\alpha$ que pasa de 0.10 a 0.20 , encontrando que el pronóstico para el mes 13 es de 7,276.41. ¿Cuál de los dos pronósticos es el más acertado?, el que en las medidas del error se encuentre más cercano al cero.

Cabe mencionar que cuando los componentes de las series de tiempo sean cíclico o estacional se puede realizar el cálculo de los pronósticos teniendo uno de promedio móvil y otro de suavización exponencial, esto con el propósito de evaluar directamente con dos métodos diferentes los datos presentados por una empresa y las medidas del error indicaran cual es el más aproximado. 
Algo más que se debe considerar es que los métodos de pronósticos no son exactos ni precisos, se deben comparar contra los hechos reales y con ello verificar que ajustes se deben realizar en los cálculos para obtener datos más confiables.

\section{Regresión Lineal}

Por último, se revisa el método de series de tiempo que se denomina regresión lineal, la cual será aplicable cuando se requiere identificar otras variables que probablemente se encuentren relacionadas con la variable de interés (Farrera Gutierrez, 2012), por ejemplo, la variable ventas se vería afectada por la variable gastos en publicidad, ya que la eficacia de la misma podría incrementar o disminuir las ventas, por lo que dicho método se considera apropiado para detectar tendencias. Con frecuencia, ésta es una consideración importante cuando se hacen pronósticos a mediano y largo plazo.

Para iniciar con este método se debe considerar la siguiente ecuación:

En donde:

$\mathrm{Y}=$ Pronóstico

$\mathrm{m}=$ Representa la pendiente de la Recta

$\mathrm{X}=$ Representa el periodo de tiempo en el cual se desea determinar el pronostico

$b=$ Representa el punto en el que la recta corta el eje vertical
Para el cálculo de cada uno de los valores de la ecuación se utilizan las siguientes formulas:

$$
m=\frac{n \Sigma x y-\Sigma x \Sigma y}{n \Sigma x^{2}-(\Sigma x)^{2}}
$$

$$
b=\frac{\Sigma y \Sigma x^{2}-\Sigma x \Sigma x y}{n \Sigma x^{2}-(\Sigma x)^{2}}
$$

Para determinar el cálculo del pronóstico a través de este método se presenta el siguiente ejemplo:

Tabla No. 4 Ejemplo de Regresión Lineal

\begin{tabular}{|c|c|c|r|}
\hline$X$ & $Y$ & & \\
\cline { 1 - 2 } Periodo & Ventas & $X^{2}$ & $X Y$ \\
\hline 1 & 10000 & 1 & $10,000.00$ \\
\hline 2 & 12000 & 4 & $24,000.00$ \\
\hline 3 & 13000 & 9 & $39,000.00$ \\
\hline 4 & 16000 & 16 & $64,000.00$ \\
\hline 5 & 19000 & 25 & $95,000.00$ \\
\hline 6 & 23000 & 36 & $138,000.00$ \\
\hline 7 & 24000 & 49 & $168,000.00$ \\
\hline 8 & 27000 & 64 & $216,000.00$ \\
\hline 9 & 29000 & 81 & $261,000.00$ \\
\hline 10 & 31000 & 100 & $310,000.00$ \\
\hline 11 & 35000 & 121 & $385,000.00$ \\
\hline 12 & 38000 & 144 & $456,000.00$ \\
\hline 13 & 39000 & 169 & $507,000.00$ \\
\hline 14 & 42000 & 196 & $588,000.00$ \\
\hline 15 & 45000 & 225 & $675,000.00$ \\
\hline 16 & 47000 & 256 & $752,000.00$ \\
\hline 136 & 450,000 & 1,496 & $4,688,000$ \\
\hline
\end{tabular}

Fuente: Elaboración Propia

En la primera etapa se deben identificar los valores que aparecen en la 
Tabla número 4. En la columna 1 aparecen los periodos de tiempo que se denominará $\mathrm{X}$, la columna 2 representa las ventas con $\mathrm{Y}$, adicionalmente la tercera columna el valor de $\mathrm{X}$ se eleva al cuadrado y por último la columna 4 representa los valores obtenidos de eliminar la columna $\mathrm{X}$ por la columna Y.

Los datos presentados en la Tabla número 4 representa un componente de tendencia como se muestra a continuación.

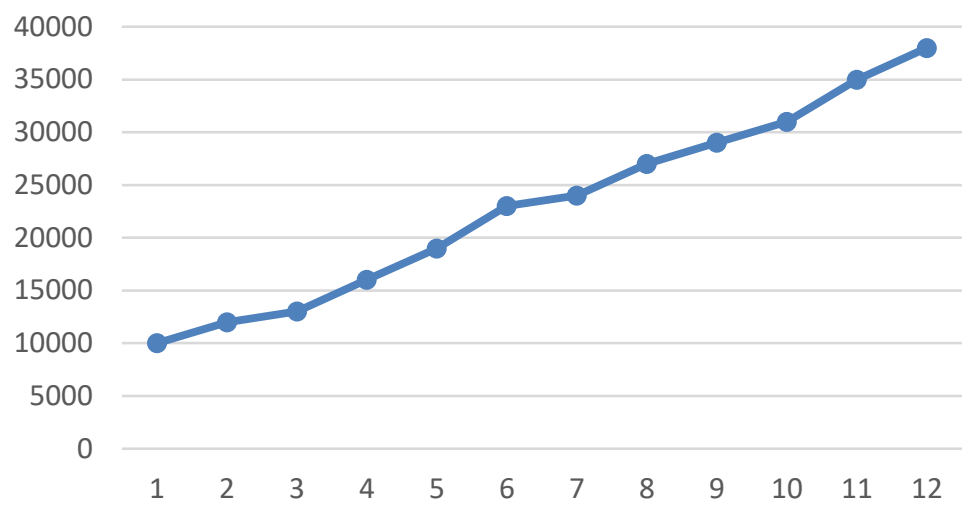

Grafica No. 6 Comportamiento de los datos de la Tabla No. 4

Fuente: Elaboración Propia

El procedimiento para este método inicia con la sumatoria de cada una de las columnas que conforman la tabla número 4 , en el caso de la columna 3 se eleva al cuadrado el valor de $\mathrm{X}$ y en la columna 4 se multiplica el valor de X por Y. Los resultados son los siguientes: Columna 1 generó una sumatoria de 136, columna 2 obtuvo un resultado de 450,000, Columna 3 alcanzó un total de 1,496, por último, la cuarta Columna generó una sumatoria de $4,688,000$. Estos datos se sustituyen en las fórmulas de $\mathrm{m} \mathrm{y} b$, generando $\mathrm{los}$ siguientes resultados:

$$
\begin{aligned}
& \mathrm{m}=2,538.24 \\
& \mathrm{~b}=6,550
\end{aligned}
$$

Por lo tanto, la ecuación de Y queda de la siguiente manera:

$$
Y=2,538.23529 x+6,550
$$

Si el pronóstico que se desea calcular es del mes 17 el pronóstico sería el siguiente:

$$
\begin{gathered}
\mathrm{Y}=2,538.23529(17)+6,550 \quad \mathrm{Y}= \\
43,150.08+6,550 \quad \mathrm{Y}=49,699.99
\end{gathered}
$$

Por aproximación 49,700.

En este método solo se sustituye el número del periodo que se desea calcular en la formula y se genera el pronóstico correspondiente.

Una vez expuesta y analizada la información sobre los diferentes modelos de pronósticos para la toma de decisiones en las mipymes; el administrador será capaz de seleccionar aquel método eficaz que le permita pronosticar el rumbo futuro de los eventos sobre los que es vulnerable; recordar que se deberá apoyar en datos históricos en función de los diferentes componentes de las series de tiempos, como se ha explicado anteriormente.

\section{Conclusiones}

Para los empresarios al dirigir una micro, pequeña o mediana empresa, pronosticar es complicado, sobre todo cuando se desconoce la importancia del tema y de las bondades de las matemáticas aplicadas al cálculo de los pronósticos, esto se debe a que la mayoría de las situaciones en donde se toma decisiones sobre el rumbo de la organización, se realiza mediante juicios anteponiendo la 
experiencia y no se apoyan en datos o hecho reales, es decir, la toma de decisiones sobre el pronóstico de eventos futuros suele ser subjetivo y con ello no se cumplen los objetivos trazados por la organización.

Dicho artículo resume el análisis de la información recabada, misma que informa al empresario de lo importante que es pronosticar y con ello tomar decisiones apoyándose sobre los diferentes métodos y técnicas de pronósticos, por lo que el objetivo de esta investigación se cumple al dar a conocer dichos modelos, mismos que le ayudarán a quien toma las decisiones, a predecir de manera sistemática las decisiones sobre las que se apoyan las actividades operativas de la organización.

Para aplicar un método de pronósticos, es fundamental contar con serie de datos que permitirán determinar aquel método cercano al futuro deseado, considerando el error que pudiera presentarse, mismo que será medido por las diferentes técnicas acercando al valor exacto del error cero, como ya se explicó, mismo que indicará las estimaciones reales.

La información descrita ha mostrado los diversos elementos que deben considerarse para el cálculo de pronósticos en los diferentes tipos de organizaciones, cuyas variables de tamaño, giro y comercialización, influyen de manera importante en la toma de decisiones para cualquier tipo de organización, por lo que al determinar el método más conveniente coadyuva al desarrollo económico futuro de las mismas.
Finalmente, el contexto global en el que se desenvuelven las micro, pequeñas y medianas empresas, es muy cambiante, siendo las variables externas como la economía, la política, la tecnología, y otras que no puede controlar, le implica poder adaptarse para enfrentar las situaciones que repercuten directamente en sus operaciones de comercialización, producción, finanzas y otras áreas que requieren vital atención, por lo que ésta primera investigación da oportunidad a integrar otro tipo de modelos apoyados en las matemáticas que contribuya a actualizar los expuestos así como denotar la importancia de los mismos para la toma de decisiones.

\section{Referencias}

Charles, G. (2003). Métoos Cuantitativos Para La Toma de Decisiones en Administración. México: McGraw Hill.

Farrera Gutierrez, A. (2012). Manual de Pronósticos Para La Toma de Decisiones. México: Digital Tecnológico de Monterrey.

Fernanda, V. (Septiembre de 2016). Universidad Nacional del Sur Argentina. Recuperado el Mayo de 2018, de http://www.matematica.uns.edu.ar/uma2016/material /Introduccion_a_los_Modelos_de_Pronosticos.pdf

J, L. (2002). El Método Delphi. Una Técnica de Previsión del Futuro. España: Ariel.

Mendoza Ramírez, M. (2010). Pronósticos y Estadísticas Para la Administración. México: ITAM.

Nuño de León, P. d. (25 de abril de 2018). Bibliotecas Digitales. Obtenido de http://www.aliat.org.mx/BibliotecasDigitales/Hospita lidad/Administracion_de_pequenas_empresas.pdf

Wilson, J., \& Koerber, D. (1992). Combining Subjective and Objective Forescasts Improves Results. Journal of Business Forecasting , 11(3), 1216. 